\title{
Combination of transurethral resection of the prostate and flexible and rigid ureteroscopy for benign prostatic hyperplasia and ureteral calculus
}

\author{
Kunlun He, Yan Liu, Dong Li, Qian Yu \\ Department of Urology Surgery, Hengshui People's Hospital, Hengshui, China \\ Contributions: (I) Conception and design: Q Yu; (II) Administrative support: Q Yu; (III) Provision of study materials or patients: D Li, Y Liu; (IV) \\ Collection and assembly of data: K He; (V) Data analysis and interpretation: K He; (VI) Manuscript writing: All authors; (VII) Final approval of \\ manuscript: All authors. \\ Correspondence to: Qian Yu. Department of Urology Surgery, Hengshui People’s Hospital, Hengshui 053000, China. Email: yuqian05041019@163.com.
}

Background: Combination of transurethral resection of the prostate (TURP) and flexible and rigid ureteroscopy (URS/RIRS) is a successful approach for patients with benign prostatic hyperplasia (BPH) and ureteral calculus (UC), and the sequence is URS/RIRS followed by TURP. This research aims to compare TURP followed by URS/RIRS with URS/RIRS followed by TURP in terms of clinical efficiency and safety. Methods: From June 2009 to June 2021, 173 patients with BPH and upper urinary tract stones were recruited through the Harrison International Peace Hospital and were divided into intervention (TURP followed by URS/RIRS) and control groups (URS/RIRS followed by TURP). We collected demographic data, primary outcomes including urinary function, and secondary outcomes including surgical parameters and complications. SPSS 21.0 was used to analyze data.

Results: When comparing the surgical parameters, the intervention group showed better results than the control group regarding surgery time and length of hospitalization. When comparing urinary function and complications, there were no differences between the intervention and control groups.

Conclusions: Although the intervention of TURP followed by URS/RIRS had similar clinical effects compared with URS/RIRS followed by TURP in the control group; the intervention saves surgery time, and decreases the length of stay and medical costs. It may therefore be a good choice for patients with BPH and UC.

Keywords: Transurethral resection of the prostate (TURP); flexible and rigid ureteroscopy (URS/RIRS); benign prostatic hyperplasia (BPH); ureteral calculus (UC)

Submitted May 25, 2021. Accepted for publication Jul 06, 2021.

doi: $10.21037 / \mathrm{tau}-21-523$

View this article at: https://dx.doi.org/10.21037/tau-21-523

\section{Introduction}

Benign prostatic hyperplasia (BPH) combined with ureteral calculus (UC) is common in middle-aged and older males, and its incidence increases with age $(1,2)$. BPH can increase the feeling of pressure on the bladder and urethral outlet, causing clinical symptoms such as frequent urination, urgency, and even incontinence, as well as hematuria (3). UC is mainly distributed in the ureteral stenosis, and about $70 \%$ is located in the lower part of the ureter. It can cause renal colic, hematuria and frequent urination (4). If the stone is obstructed, it can also cause serious complications such as hydronephrosis and renal insufficiency. In addition, $\mathrm{BPH}$ combined with UC impairs patients' sexual life and sexual functioning $(5,6)$.

For BPH treatment combined with UC, transurethral resection of the prostate (TURP) and ureteroscopy combined with holmium laser lithotripsy are commonly used $(7,8)$. The combination of the two treatments can 


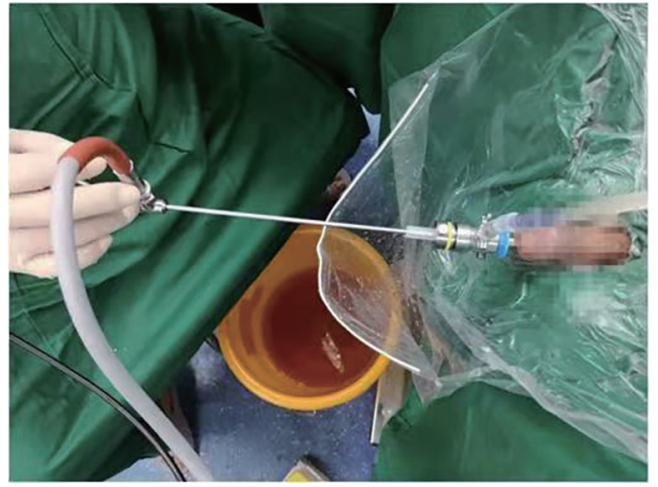

Figure 1 Ureteroscopy was inserted into the bladder through the sheath of the resectoscope.

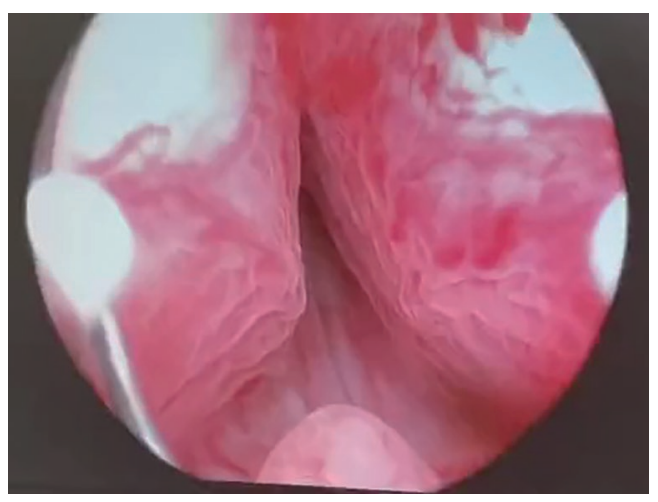

Figure 2 Observation of prostate gland by electrotomy.

usually achieve better results. TURP uses a ring electrode to emit two different waveforms of high-frequency currents for cutting and electrocoagulation (9). Compared with traditional open surgery, TURP has the advantages of less trauma and faster recovery. It can effectively increase maximum flow rate (Qmax) by about $162 \%$, reduce international prostate symptom score (IPSS) score by $70 \%$ on average, and reduce post-void residual urine volume (PVR) by about $77 \%$. Its shortcomings are incomplete gland resection, poor hemostatic effect, intraoperative transurethral resection syndrome (TURS), and retrograde ejaculation after surgery (10).

Ureteroscopic lithotripsy is the primary method for complicated upper ureteral calculi (Figure 1). The ureteroscope used can be divided into two types: rigid and flexible. The flexible ureteroscope has a flexible body and a small tube diameter and is operated with a rigid lens (11). Rigid ureteroscopy is also a commonly used surgical procedure.
Its advantages are convenient operation, better expansion of the ureter, and reduced operation time (12). Nowadays, the widely used method in clinical practice is flexible and rigid ureteroscopy (URS/RIRS) for holmium laser lithotripsy. The technique involves the insertion of the ureteroscope into the ureter to locate the stones and using the holmium laser to break down the stones appropriately (13) (Figure 2).

In the past, simultaneous intracavitary surgery was carried out to treat ureteral calculi combined with BPH, and now the treatment plan was also used with ureteral rigid and flexible mirror holmium laser to crush the calculi. Surgeons placed a double-J tube and performed TURP surgery, which can reduce the operating time. These approaches improve the efficiency of surgery and achieve good results (14). Based on previous research, we found that if the surgery sequence is adjusted during the period of intracavitary surgery, the current TURP surgery and the ureteral rigid and flexible mirror holmium lasers may have a better effect. Therefore, we collected data on several cases to evaluate a new surgical approach, whereby URS/RIRS follows TURP compared with previous simultaneous intracavitary surgery (URS/ RIRS followed by TURP). The novelty of this research is that we changed the sequence of URS/RIRS and TURP. Since previous researches conducted URS/RIRS first and then carry out TUPR, we use TURP first and then use URS/RIRS. We present the following article in accordance with the STROBE reporting checklist (available at https:// dx.doi.org/10.21037/tau-21-523).

\section{Methods}

\section{Design of the study}

From June 2009 to June 2021, 173 patients with BPH and upper urinary tract stones were recruited through the Harrison International Peace Hospital. The age of the patients ranged from 55 to 86 years old. The primary purpose of this study was to explore the primary outcomes, including urinary function and secondary outcomes, including surgical parameters, between our surgical approach (intervention group) and the previous surgical approach (control group). The secondary purpose was to evaluate the incidence of complications such as postoperative fever and urinary tract infection to explore the safety of our surgical plan. We also collected demographic data, including age, body mass index (BMI), and stone diameter. All procedures performed in this study involving human participants were in accordance with the Declaration of Helsinki (as revised in 
2013). The study was approved by regional ethics board of Hengshui People's Hospital (No.: HS600325) and informed consent was taken from all the patients.

\section{Patients}

Case selection criteria followed the 2014 Department of Urology guidelines for prostatic hyperplasia operations: (I) patients with urinary retention, who cannot urinate after removal of the catheter; (II) patients with repeated gross hematuria; (III) patients with renal insufficiency; (IV) patients with bladder stones; (V) patients with recurrent urinary tract infection; (VI) patients with giant bladder diverticulum; (VII) the diameter of the upper urinary tract stones is less than or equal to $2 \mathrm{~cm}$, and patients' are older than 55 years; and (VIII) no cardiopulmonary disease and neurogenic bladder patients.

The exclusion criteria were: (I) congenital malformation of ureter; (II) urethral stricture; (III) acute urethritis; (IV) patients with severe cardiopulmonary disease; and (V) patients with coagulation dysfunction.

\section{Procedures}

From June 2009 to March 2016, we selected 73 cases who underwent URS/RIRS first and then received TURP for inclusion in the control group, and from March 2016 to June 2021, 100 patients were selected who underwent TURP first and then received URS/RIRS for inclusion in the intervention group. In the whole process, patients, patients in the intervention group and the control group followed the principle of randomization.

For the control group, we carried out the following treatment: after administration of anesthesia and routine disinfection, an 8.0/8.9 $\mathrm{F}$ rigid ureteroscope (Wolf, Germany) was inserted into the bladder through the urethra, a zebra guidewire was inserted into the ureter using a ureteroscope, and the zebra guide wire-guided ureteroscope was inserted into the ureter to find the ureteral calculi. A Holmium Laser Fiber (Dornier, Germany) was inserted into the ureteral calculi through the ureteroscope. We gradually broke down the calculi, and then a double-J tube was inserted into the ureter. Then, the ureteroscope was removed, and a resectoscope (Smir, China) Smir plasma resectoscope was placed under direct vision through the urethra. The hyperplastic gland was resected up to the surgical capsule both clockwise and anticlockwise. Finally, we trimmed the tip of the prostate. After sufficient hemostasis, the removed tissue was washed out and sent for pathological examination. If there was no apparent residual gland, a three-way F22 cavity irrigation catheter was placed there.

The intervention group was treated as follows: after administration of anesthesia, patients on the operating table assumed the lithotomy position. Following TURP, the removed tissue was washed out, and we detached the handle of the resectoscope. We placed the disposable irrigator in the sheath of the resectoscope. An 8.0/8.9 F rigid ureteroscope (Wolf, Germany) was placed inside the hose, and the bladder was reached through the sheath of the resectoscope. The guidewire was inserted into the affected ureter, and the stones were crushed with a Holmium Laser Fiber (Dornier, Germany). Then a double-J tube was inserted into the ureter to remove the stones. The ureteroscope was withdrawn, and we inserted the handle of the resectoscope once again. The glandular fossa was observed until it stopped bleeding entirely. A three-way F22 cavity irrigation catheter was placed there to remove the stones. If the stone was located in the renal pelvis, the zebra guidewire was inserted into the affected side of the ureter using a rigid ureteroscope. Under the guidewire guidance, the flexible ureteroscope sheath of BOCO $12 / 14 \mathrm{~F}$ was inserted into the ureteropelvic junction. A flexible ureteroscope (Olympus P6) was inserted through a flexible ureteroscope sheath. The holmium laser fiber was inserted into the stone through the adjustable ureteroscope operation channel to crush the stone gradually.

\section{Statistical analysis}

Data collection followed the requirements of prospective intervention research. Patients were given verbal information, including information materials published by the Chinese Urology Association. Data relating to continuous variables are reported as means $\pm \mathrm{SD}$, and those on categorical variables as frequencies. SPSS 21.0 was used for statistical analysis, and student $t$-tests analyzed continuous variables, and non-parameters by KruskalWallis tests or Mann U Whitney tests. For the categorical variables, chi-square and Fisher precision tests were conducted. The significance level was set at $\mathrm{P}<0.05$.

\section{Results}

\section{Baseline patient characteristics}

One hundred and seventy-three patients were enrolled. 
Table 1 Demographic and clinical characteristics of each study group

\begin{tabular}{|c|c|c|c|c|}
\hline Variables & Intervention $(n=100)$, mean \pm SD & Control $(n=73)$, mean $\pm S D$ & t value & $P$ value \\
\hline BMI $\left(\mathrm{kg} / \mathrm{m}^{2}\right)$ & $24.55 \pm 4.32$ & $24.36 \pm 4.51$ & 0.29 & 0.77 \\
\hline Mean ureteral stone diameter (mm) & $7.72 \pm 2.63$ & $7.25 \pm 2.36$ & 1.23 & 0.22 \\
\hline
\end{tabular}

BMI, body mass index.

Table 2 Surgical parameters of each study group

\begin{tabular}{|c|c|c|c|c|}
\hline Variables & Intervention $(n=100)$, mean \pm SD & Control $(n=73)$, mean \pm SD & $\chi^{2} / \mathrm{t}$ value & $P$ value \\
\hline Operation time (min) & $113.23 \pm 12.54$ & $118.52 \pm 14.63$ & -2.49 & 0.01 \\
\hline Length of hospitalization (day) & $8.28 \pm 1.54$ & $9.99 \pm 1.22$ & -8.14 & $<0.01$ \\
\hline
\end{tabular}

The intervention group received TURP followed by URS/ RIRS, and the control group received URS/RIRS followed by TURP. As shown in Table 1, preoperative age, BMI, and average UC diameter of the two groups were comparable, and there were no statistically significant differences.

\section{Patient surgical parameters}

As shown in Table 2, there were no statistically significant differences in the success rate of lithotripsy between the intervention and the control group $(\mathrm{P}>1)$. The average operating time of the intervention group was $113.23 \pm 12.54$ vs. $118.52 \pm 14.63$ minutes in the control group, which is a statistically significant difference $(\mathrm{P}=0.01)$. The intervention group was less than the control group in the success rate of lithotripsy. The average hospital stay was $8.28 \pm 1.54$ and $9.99 \pm 1.22$ days in the intervention and control group, respectively, indicating a statistically significant difference indicating superiority of the intervention group $(\mathrm{P}=0.01$ or $\mathrm{P}<0.01)$.

\section{Urinary function parameters}

Treatment efficacy [IPSS, quality of life (QOL), Qmax, and PVR] as the primary outcome and IPSS, QOL, Qmax, and PVR at 3 months are listed in Table 3. IPSS, QOL, Qmax, and PVR after 3 months showed no significant differences between the two groups before and after surgery.

\section{Complication parameters}

Complications in the two groups are presented in Table 4. The incidence of intraoperative complications, including TURS and blood transfusion, were not significantly different. Regarding late complications, including fever, urinary tract infection, urinary retention, urethral strictures, and recurrence, no significant differences were observed between the two groups $(\mathrm{P}>0.05)$.

\section{Discussion}

TURP has become the first choice for endovascular surgery to treat $\mathrm{BPH}$ based on years of clinical experience. Rigid/flexible ureteroscopic holmium laser lithotripsy has the advantages of high safety, high success rate, limited complications, and little intraoperative bleeding $(15,16)$. However, for some patients with pronounced elevation of the bladder neck or obvious protruding of the middle lobe of the prostate into the bladder, the ureteroscope may not successfully insert into the ureter, or it may be difficult to ascend after it has been inserted into the ureter and cause lithotripsy failure $(17,18)$. In patients with BPH and UC, first, the ureteroscope is inserted to crush the upper urinary tract stones, and then the double-J tube is indwelled, followed by withdrawal of the ureteroscope. The plasma resectoscope is used again for TURP, but the frequency of plasma resectoscope applied increases. The chance of 
Table 3 Urinary function parameters of each study group before and after surgery

\begin{tabular}{|c|c|c|c|c|}
\hline Variables & Intervention $(n=100)$, mean \pm SD & Control $(n=73)$, mean \pm SD & t value & $P$ value \\
\hline IPSS & $25.48 \pm 2.65$ & $25.37 \pm 3.25$ & 0.24 & 0.81 \\
\hline QOL & $4.82 \pm 0.75$ & $4.63 \pm 0.74$ & 1.66 & 0.09 \\
\hline Qmax & $6.67 \pm 1.88$ & $7.15 \pm 2.16$ & -1.52 & 0.13 \\
\hline \multicolumn{5}{|c|}{ Postoperative after 3 months } \\
\hline IPSS & $5.01 \pm 1.95$ & $5.25 \pm 1.82$ & -0.83 & 0.41 \\
\hline QOL & $1.23 \pm 0.92$ & $1.35 \pm 0.82$ & -0.90 & 0.37 \\
\hline Qmax & $18.13 \pm 2.18$ & $18.72 \pm 2.52$ & -1.61 & 0.11 \\
\hline
\end{tabular}

IPSS, international prostate symptom score; QOL, quality of life; Qmax, maximum flow rate; PVR, post-void residual urine volume.

Table 4 Complications of each study group

\begin{tabular}{|c|c|c|c|c|}
\hline Variables & Intervention $(n=100)$ & Control $(n=73)$ & $\chi^{2}$ value & $P$ value \\
\hline TURS & 1 & 2 & 0.08 & 0.78 \\
\hline Blood transfusion & 2 & 2 & 0.01 & 1 \\
\hline Fever & 3 & 7 & 0.23 & 0.64 \\
\hline \multicolumn{5}{|c|}{ Postoperative complications, $\mathrm{n}$} \\
\hline Urinary retention & 1 & 1 & 0.01 & 1 \\
\hline Urethral strictures & 1 & 2 & 0.01 & 1 \\
\hline Recurrence & 1 & 1 & 0.01 & 1 \\
\hline
\end{tabular}

TURS, transurethral resection syndrome.

urethral mucosal damage is significantly increased after surgery. When the ureteral calculi are crushed, the double-J tube is placed before TURP is performed $(19,20)$. There is continuous high pressure in the bladder, and the chance of the bladder leaking fluid into the renal pelvis retrogradely increases. The risk of retrograde infection of the renal pelvis is also significantly increased. In addition, persistent high pressure in the renal pelvis increases renal dysfunction and the risk of bacteremia caused by infection of the renal pelvis and urine flowing back into the blood $(21,22)$. Therefore, when following this new surgical approach, it is imperative to avoid the above risks.

First of all, although this study also applies a resectoscope and rigid ureteroscope/flex scope to treat prostate hyperplasia and upper urinary tract stones, the sequence of surgery is reversed. TURP can change the state of the cutting electrode and increase the current generato. At the same time, the operating area is clear, and the positioning of stone is accurate. It can fully display the anatomical position and size of the prostate, and the resection tissue is delicate. The prostate apex is accurately trimmed without obturator nerve reflexes and other symptoms. In addition, the operation is convenient for hemostasis, patients recover quickly after the process, injury is minor, and most patients tolerate the procedure well. Then, the rigid/ soft ureteroscope is inserted into the ureter through the 
resection scope sheath, which can effectively avoid the risks associated with previous simultaneous surgical methods. Secondly, the combined application of a resectoscope and rigid/soft ureteroscope in this study can prevent damage to the urethral mucosa caused by multiple procedures. The treatment of prostate hyperplasia also treats upper urinary tract stones, shortens the operating time, reduces surgical trauma, decreases the average hospital stay of patients, and can effectively reduce patients' medical expenses. Finally, in terms of therapeutic effects, compared with the traditional simultaneous surgical approach, the surgical procedure used in this study shows no statistically significant differences in the success rate of lithotripsy, treatment efficiency indicators, and so on. In terms of treatment safety, the comparisons of intraoperative complications and postoperative complications at 3 months also showed no statistically significant differences between the experimental and control group.

At present, there are few studies on the combination of TURP and rigid/flexible ureteroscopy for $\mathrm{BPH}$ with ureteral calculi, which means that we cannot compare our results with those of other researchers. We hope that more researchers will test this protocol in the future. In the surgery process, we suggest that if it isn't accessible to indwelling catheters after TURP, and the metal guidewire is usually used first. If it doesn't work, our standard method is to put the zebra guide wire into the bladder and then exit the mirror.

In summary, if TURP occurs before URS/RIRS, operating and hospitalization times decrease compared with URS/RIRS before TURP, thereby saving medical resources and expenses. However, there is not much difference between the two surgical options in treatment efficacy and surgical safety. We believe that TURP followed by URS/ RIRS is a good choice for BPH with UC and that it is a surgical approach that deserves further analysis and is worth promoting.

\section{Acknowledgments}

Funding: None.

\section{Footnote}

Reporting Checklist: The authors have completed the STROBE reporting checklist. Available at https://dx.doi. org/10.21037/tau-21-523

Data Sharing Statement: Available at https://dx.doi. org/10.21037/tau-21-523

Conflicts of Interest: All authors have completed the ICMJE uniform disclosure form (available at https://dx.doi. org/10.21037/tau-21-523). The authors have no conflicts of interest to declare.

Etbical Statement: The authors are accountable for all aspects of the work in ensuring that questions related to the accuracy or integrity of any part of the work are appropriately investigated and resolved. All procedures performed in this study involving human participants were in accordance with the Declaration of Helsinki (as revised in 2013). The study was approved by regional ethics board of Hengshui People's Hospital (No.: HS600325) and informed consent was taken from all the patients.

Open Access Statement: This is an Open Access article distributed in accordance with the Creative Commons Attribution-NonCommercial-NoDerivs 4.0 International License (CC BY-NC-ND 4.0), which permits the noncommercial replication and distribution of the article with the strict proviso that no changes or edits are made and the original work is properly cited (including links to both the formal publication through the relevant DOI and the license). See: https://creativecommons.org/licenses/by-nc-nd/4.0/.

\section{References}

1. Wagrell L, Schelin S, Nordling J, et al. Feedback microwave thermotherapy versus TURP for clinical BPH-a randomized controlled multicenter study. Urology 2002;60:292-9.

2. Lokeshwar SD, Harper BT, Webb E, et al. Epidemiology and treatment modalities for the management of benign prostatic hyperplasia. Transl Androl Urol 2019;8:529-39.

3. Ding H, Du W, Lu ZP, et al. Photoselective green-light laser vaporisation vs. TURP for BPH: meta-analysis. Asian J Androl 2012;14:720-5.

4. Wang J. Feasibility study of endoscopic simultaneous treatment of ureteral calculi and benign prostatic hyperplasia. Shanxi Medical Journal 2015;44:2116-9.

5. Ye L, Shen Y, Hou X, et al. Causes and management of postoperative massive bleeding of $\mathrm{BPH}$ patients treated with TURP (report of 55 cases). Journal of Clinical Urology 2005;20:27-9.

6. Jichlinski P, Oswald M, Schmidlin FR, et al. Combination of thermocoagulation and vaporization using an $\mathrm{Nd}$ : 
YAG/KTP laser versus TURP in BPH treatment: results of a multicenter prospective study. In: Lasers in surgery: advanced characterization, therapeutics, and systems VIII. International Society for Optics and Photonics, 1998;3245:85-9.

7. Wang B, Zhang S, Sun C, et al. Comparison between a transurethral prostate split and transurethral prostate resection for benign prostatic hyperplasia treatment in a small prostate volume: a prospective controlled study. Ann Transl Med 2020;8:1016.

8. Goldberg H, Holland R, Tal R, et al. The impact of retrograde intrarenal surgery for asymptomatic renal stones in patients undergoing ureteroscopy for a symptomatic ureteral stone. J Endourol 2013;27:970-3.

9. Gravenstein D. Transurethral resection of the prostate (TURP) syndrome: a review of the pathophysiology and management. Anesth Analg 1997;84:438-46.

10. Wagrell L, Schelin S, Nordling J, et al. Three-year followup of feedback microwave thermotherapy versus TURP for clinical BPH: a prospective randomized multicenter study. Urology 2004;64:698-702.

11. Hollenbeck S, Newton A, Ranjbar K, et al. JSON Responses to RESTful URL Queries for RIRs and DNRs. 2012. Available online: https://datatracker.ietf.org/doc/ draft-newton-weirds-unified-json-response/

12. Pan J, Chen Q, Xue W, et al. RIRS versus mPCNL for single renal stone of $2-3 \mathrm{~cm}$ : clinical outcome and costeffective analysis in Chinese medical setting. Urolithiasis 2013;41:73-8.

13. Alkan E, Arpali E, Ozkanli AO, et al. RIRS is equally efficient in patients with different BMI scores. Urolithiasis 2015;43:243-8.

14. Ferretti S, Campobasso D, Bocchialini T, et al. Results and complications of retrograde approach (URS/RIRS) in pediatric urolithiasis: a multicentric experience. Eur Urol
Suppl 2019;18:e3212.

15. Persu C, Georgescu D, Arabagiu I, et al. TURP for BPH. How large is too large? J Med Life 2010;3:376-80.

16. Gilling P. TURP remains a safe and effective alternative for benign prostatic hyperplasia (BPH) surgery. BJU Int 2014;113:5-6.

17. Wilhelm K, Hein S, Adams F, et al. Ultra-mini PCNL versus flexible ureteroscopy: a matched analysis of analgesic consumption and treatment-related patient satisfaction in patients with renal stones $10-35 \mathrm{~mm}$. World J Urol 2015;33:2131-6.

18. Grosse G, Kalvius G M, Kratzer A, et al. Uranium moment collapse in magnetically diluted US. J Magn Magn Mater 1999;205:79-89.

19. Nicholson TM, Moses MA, Uchtmann KS, et al. Estrogen receptor- $\alpha$ is a key mediator and therapeutic target for bladder complications of benign prostatic hyperplasia. J Urol 2015;193:722-9.

20. Parliteanu BG, Manu $M$, Hurduc V, et al. Is prostatic endoscopic surgery a "must" after stone endoscopic removal in patients with $\mathrm{BPH}$ and bladder lithiasis? Eur Urol Suppl 2018;17:e2648.

21. Wang LQ, Pu HM, Yan YJ, et al. A clinical study on treating $\mathrm{BPH}$ and bladder complicated stone with minor incision operation plus TURP under direct vision (report of 40 cases). China Journal of Endoscopy 2006; 12:242-3, 246.

22. Bachmann A, Schürch L, Ruszat R, et al. Photoselective vaporization (PVP) versus transurethral resection of the prostate (TURP): a prospective bi-centre study of perioperative morbidity and early functional outcome. Eur Urol 2005;48:965-71; discussion 972.

(English Language Editors: B. Meiser and J. Chapnick)
Cite this article as: $\mathrm{He} \mathrm{K}$, Liu Y, Li D, Yu Q. Combination of transurethral resection of the prostate and flexible and rigid ureteroscopy for benign prostatic hyperplasia and ureteral calculus. Transl Androl Urol 2021;10(8):3395-3401. doi: 10.21037/ tau-21-523 